\title{
An improved nonperturbative method for studying two-dimensional vortex liquids
}

\author{
J Yeo, H Park and S Yi \\ Department of Physics, Konkuk University, Seoul 143-701, Korea
}

Received 27 December 2005, in final form 3 March 2006

Published 30 March 2006

Online at stacks.iop.org/JPhysCM/18/3607

\begin{abstract}
We show how a systematic improvement can be made on the nonperturbative parquet approximation method which was previously used to study the effect of thermal fluctuations in vortex liquids in high temperature superconductors. This is achieved by including an infinite subset of Feynman diagrams contributing to the renormalized four-point vertex function of the Ginzburg-Landau model, which was omitted in the original approximation. We find that the growing crystalline order in the vortex liquid is more pronounced in the improved approximation. In particular, the second and third peaks in the liquid structure factor, which appeared as one peak in the original approximation, are now resolved.
\end{abstract}

\section{Introduction}

Thermal fluctuations play a more important role in high temperature superconductors than in conventional materials because of strong anisotropy, high temperature, and short coherence length [1]. Therefore, a high temperature superconductor in a magnetic field is believed to be in a vortex liquid phase resulting from the melting of the Abrikosov vortex lattice predicted by the mean field theory [2]. For bulk materials, experiments [3-5] detect well below the upper critical field $H_{\mathrm{c} 2}$ sharp drops in resistivity and steps in the magnetization and the specific heat, which are interpreted when the strength of disorder is weak as being due to a vortex liquid undergoing a first-order phase transition into presumably the Abrikosov lattice as the temperature is lowered. However, the situation is more complicated because the first-order transition disappears at both high and low magnetic fields [3, 6-8].

For two-dimensional superconducting films, the question of whether the vortex liquid undergoes a phase transition at all into the low temperature ordered state is still controversial. The perturbation expansions around the high temperature liquid state using the GinzburgLandau (GL) model within the lowest Landau level (LLL) approximation have been carried out to find evidence of the transition to the ordered phase at low temperatures [9, 10]. A weak firstorder transition is detected in two-dimensional vortex liquids from numerical simulations [11]. However, numerical simulation results depend strongly on the boundary conditions as the 
Monte Carlo simulation performed on a spherical geometry [12] shows no sign of a finite temperature transition.

As a nonperturbative analytic (i.e. nonsimulational) approach, the parquet approximation has been successfully applied to a two-dimensional vortex system [13] and also to vortices in a layered superconductor $[14,15]$. It is free from any finite size effect perpendicular to the field direction and sophisticated enough to capture the growing crystalline order developing in the vortex liquid as the temperature is lowered. The parquet approximation deals with the renormalized four-point function of the vortex system which is obtained by summing an infinite subset of Feynman diagrams, the so-called parquet diagrams. Although the parquet diagrams seem to form a minimal set of Feynman diagrams which capture the growing crystalline order properly, there is no a priori reason to neglect the non-parquet diagrams. This is because there is no apparent small parameter associated with the non-parquet diagrams. In this paper, we present a first attempt to go beyond the parquet approximation. We show how one can include systematically the non-parquet diagrams into the existing nonperturbative calculations. We do this by devising a way to take into account yet another infinite subset of Feynman diagrams which are omitted in the previous calculations. This procedure is similar to what one does in the integral equation approach to ordinary liquids [16], where integral equations such as the hypernetted chain equation are improved by adding an appropriate set of diagrams. We shall see that the growing crystalline order developing in the vortex liquid as the temperature decreases is more pronounced when the non-parquet diagrams are included, although no finite temperature phase transition is detected as in the previous studies [13].

In the next section, we briefly set up the parquet diagram decomposition method for the two-dimensional vortex liquid systems. In section 3, we show how the non-parquet contributions can be included in the formulation. The improved integral equations which include these new diagrams are solved numerically in section 4 . We conclude with discussion in section 5 .

\section{Parquet graph resummation method}

The parquet graph resummation method for vortex liquids [13] is based on the LLL approximation of the GL model for a superconductor in a perpendicular magnetic field. For a superconducting film, the GL free energy with the order parameter denoted by $\Psi$ is given by

$$
F[\Psi]=\int \mathrm{d}^{2} \mathbf{r}\left[\alpha|\Psi(\mathbf{r})|^{2}+\frac{\beta}{2}|\Psi(\mathbf{r})|^{4}+\frac{1}{2 m}\left|\left(-\mathrm{i} \hbar \nabla-\frac{e^{*}}{c} \mathbf{A}\right) \Psi\right|^{2}\right],
$$

where $\alpha, \beta$, and $m$ are phenomenological parameters and $e^{*}=2 e$. We take $\mathbf{B}=\nabla \times \mathbf{A}$ as constant and uniform, and use the LLL approximation, which is believed to be valid over a large portion of the vortex liquid region [17]. In the symmetric gauge, where $\mathbf{A}=\frac{B}{2}(-y, x, 0)$, the LLL wavefunction is given by $\Psi^{\operatorname{LLL}}(\mathbf{r})=\exp \left(-\mu^{2}|z|^{2} / 4\right) \phi(z)$ where $\mu^{2}=e^{*} B / \hbar c$ and $\phi(z)$ is an arbitrary analytic function of $z=x+\mathrm{i} y$. In the LLL approximation, the free energy becomes

$$
F\left[\phi, \phi^{*}\right]=\int \mathrm{d} z^{*} \mathrm{~d} z\left[\alpha_{H} \mathrm{e}^{-\mu^{2}|z|^{2} / 2}|\phi(z)|^{2}+\frac{\beta}{2} \mathrm{e}^{-\mu^{2}|z|^{2}}|\phi(z)|^{4}\right]
$$

where $\alpha_{H} \equiv \alpha+\mathrm{e}^{*} B \hbar / 2 \mathrm{~cm}$ changes sign crossing the upper critical field line $H_{\mathrm{c} 2}(T)$. The effect of thermal fluctuations in the two-dimensional vortex liquid systems is determined by the partition function $Z=\int \mathcal{D} \phi \mathcal{D} \phi^{*} \exp \left(-F\left[\phi, \phi^{*}\right]\right)$. In this section, we set up the parquet diagram resummation method and calculate various correlation functions with respect to the 

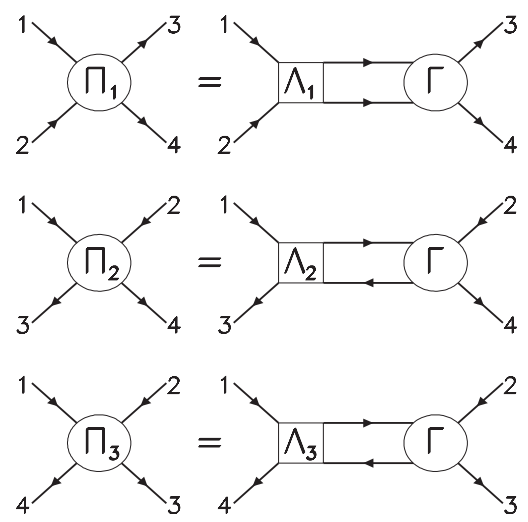

Figure 1. A graphical representation of the Bethe-Salpeter equations for the reducible parts $\Pi_{i}$. (See equation (7).)

partition function. One can develop the standard perturbation theory from the given partition function. The bare propagators are given by

$$
\left\langle\phi^{*}\left(z^{*}\right) \phi(z)\right\rangle_{0}=\frac{1}{\alpha_{H}}\left(\frac{\mu^{2}}{2 \pi}\right) \mathrm{e}^{\mu^{2} z^{*} z / 2} .
$$

Since the magnetic length $\mu^{-1}$ is the only length scale perpendicular to the field direction which appears in the propagator [13], the fully renormalized propagator can also be written in the same way as equation (3) with the renormalized $\alpha_{R}$ replacing the bare $\alpha_{H}$. The renormalized $\alpha_{R}$ is determined self-consistently in the parquet approximation as will be seen later.

The main quantity one calculates in the parquet approximation is the renormalized connected four-point function $\left\langle\phi^{*}\left(z_{1}^{*}\right) \phi^{*}\left(z_{2}^{*}\right) \phi\left(z_{3}\right) \phi\left(z_{4}\right)\right\rangle_{c}$. An important feature of the LLL approximation is that this renormalized quantity can be completely described by a single vertex function $\Gamma(\mathbf{k})$ [13], where the momentum $\mathbf{k}$ corresponds to the two-dimensional space perpendicular to the magnetic field. In general this quantity can be written as

$$
\begin{gathered}
\left\langle\phi^{*}\left(z_{1}^{*}\right) \phi^{*}\left(z_{2}^{*}\right) \phi\left(z_{3}\right) \phi\left(z_{4}\right)\right\rangle_{c}=-\frac{2 \beta}{\alpha_{R}^{4}}\left(\frac{\mu^{2}}{2 \pi}\right)^{2} \exp \left(\frac{\mu^{2}}{2}\left(z_{1}^{*} z_{3}+z_{2}^{*} z_{4}\right)\right) \int \frac{\mathrm{d} k^{*} \mathrm{~d} k}{(2 \pi)^{2}} \Gamma(\mathbf{k}) \\
\times \exp \left(-\frac{|k|^{2}}{2 \mu^{2}}-\frac{\mathrm{i}}{2}\left\{k^{*}\left(z_{3}-z_{4}\right)+k\left(z_{1}^{*}-z_{2}^{*}\right)\right\}\right) .
\end{gathered}
$$

Note that to the lowest order, $\Gamma(\mathbf{k})=\Gamma_{B}(\mathbf{k})=\exp \left(-|k|^{2} / 2 \mu^{2}\right)$ is the bare vertex.

The parquet approximation has been widely used [18] in many branches of many-body physics. For the vortex liquids, we make a resummation over all parquet diagrams by first noting that the contributions to $\Gamma$ can be decomposed into the totally irreducible part denoted by $R$ and the reducible part. The reducible part in turn can be written as the sum of three parts $\Pi_{i}(i=1,2,3)$ representing the contributions from three different channels as shown in figure 1. (A detailed discussion of the diagrammatic decomposition can be found in [13].) We have

$$
\Gamma(\mathbf{k})=R(\mathbf{k})+\sum_{i=1}^{3} \Pi_{i}(\mathbf{k}) .
$$

Each reducible vertex $\Pi_{i}$ is composed of an irreducible vertex $\Lambda_{i}$ where

$$
\Lambda_{i}(\mathbf{k})=R(\mathbf{k})+\sum_{j \neq i} \Pi_{j}(\mathbf{k})
$$


and the renormalized $\Gamma$ via the following Bethe-Salpeter equations (see figure 1):

$$
\begin{aligned}
& \Pi_{1}(\mathbf{k})=-x\left[\Lambda_{1} \circ \Gamma\right](\mathbf{k}), \\
& \Pi_{2}(\mathbf{k})=-2 x \Lambda_{2}(\mathbf{k}) \Gamma(\mathbf{k}), \\
& \Pi_{3}(\mathbf{k})=-2 x\left[\Lambda_{3} * \Gamma\right](\mathbf{k}),
\end{aligned}
$$

where the operation $\circ$ between two arbitrary functions $f(\mathbf{k})$ and $g(\mathbf{k})$ is defined by

$$
(f \circ g)(\mathbf{k})=\frac{2 \pi}{\mu^{2}} \int \frac{\mathrm{d}^{2} \mathbf{k}^{\prime}}{(2 \pi)^{2}} f\left(\mathbf{k}-\mathbf{k}^{\prime}\right) g\left(\mathbf{k}^{\prime}\right) \cos \left(\left(k_{x} k_{y}^{\prime}-k_{y} k_{x}^{\prime}\right) / \mu^{2}\right)
$$

and $f * g$ is just the convolution without the cosine factor. The parquet approximation corresponds to keeping only the bare vertex function $\Gamma_{B}(\mathbf{k})$ in the totally irreducible vertex $R(\mathbf{k})$, and neglecting all the higher order non-parquet diagrams. The lowest order of the diagrams neglected in this approximation is $\mathrm{O}\left(\beta^{4}\right)$. The main point of this paper, which will be discussed in the next section, is finding a way to incorporate systematically the diagrams neglected in the parquet approximation. In equation (7), we have used the dimensionless parameter $x=\mu^{2} \beta / 2 \pi \alpha_{R}^{2}$, which originated from the two renormalized propagator lines in $\Pi_{i}$. This parameter $x$ is determined self-consistently from the following exact Dyson equation, which relates the renormalized propagator and the renormalized quartic vertex:

$$
\alpha_{T}=\frac{1}{\sqrt{x}}\left[1-2 x+2 x^{2}\left(\frac{2 \pi}{\mu^{2}}\right) \int \frac{\mathrm{d}^{2} \mathbf{k}}{(2 \pi)^{2}} \mathrm{e}^{-k^{2} / 2 \mu^{2}} \Gamma(\mathbf{k})\right],
$$

where $\alpha_{T} \equiv \alpha_{H} \sqrt{2 \pi / \beta \mu^{2}}$ is the dimensionless temperature. We can show that $\alpha_{T}$ is proportional to $-(1-t-h) /(t h)^{1 / 2}$, where $t=T / T_{\mathrm{c} 0}$ and $h=H / H_{\mathrm{c} 2}(0)$ with $T_{\mathrm{c} 0}$ and $H_{\mathrm{c} 2}(0)$ being the critical temperature at zero field and the upper critical field at zero temperature, respectively. Note that equations (5)-(8) form a closed set of equations for $\Gamma$ (k) for given $\alpha_{T}$ and $R(\mathbf{k})$. Note also that this set of equations are exact relations for the vertex function $\Gamma(\mathbf{k})$. Recall that in the parquet approximation $R(\mathbf{k})$ is approximated as the bare vertex $\Gamma_{B}(\mathbf{k})$.

Using the solutions to the above equations we can calculate several interesting physical quantities. Among them, we focus on the structure factor, which is a measure of the correlation between vortices in a vortex liquid. It is calculated from

$$
\chi\left(\mathbf{r}-\mathbf{r}^{\prime}\right)=\left\langle|\Psi(\mathbf{r})|^{2}\left|\Psi\left(\mathbf{r}^{\prime}\right)\right|^{2}\right\rangle-\left\langle|\Psi(\mathbf{r})|^{2}\right\rangle\left\langle\left.\Psi\left(\mathbf{r}^{\prime}\right)\right|^{2}\right\rangle .
$$

The structure factor $\Delta(\mathbf{k})$ used in this paper is then defined as

$$
\Delta(\mathbf{k}) \equiv\left(\frac{2 \pi \alpha_{R}^{2}}{\mu^{2}}\right) \mathrm{e}^{\mathbf{k}^{2} / 2 \mu^{2}} \int \mathrm{d}^{2} \mathbf{R} \mathrm{e}^{\mathrm{ik} \cdot \mathbf{R}} \chi(\mathbf{R}) .
$$

By joining two external legs of the four-point correlation functions in (4), we obtain a simple relation,

$$
\Delta(\mathbf{k})=1-2 x \Gamma(\mathbf{k})
$$

The above coupled integral equations for $\Gamma(\mathbf{k})$ can be solved numerically. In [13], the parquet equations were solved for two-dimensional vortex liquids with and without quenched impurities. The parquet equations can also be solved for the vortices in layered superconductors [14].

\section{Non-parquet contributions}

In this section we discuss how the contributions from the non-parquet diagrams can be included into the correlation functions of the two-dimensional vortex liquid. Recall that, in the parquet approximation, all the diagrams contributing to the totally irreducible vertex $R(\mathbf{k})$ are neglected 


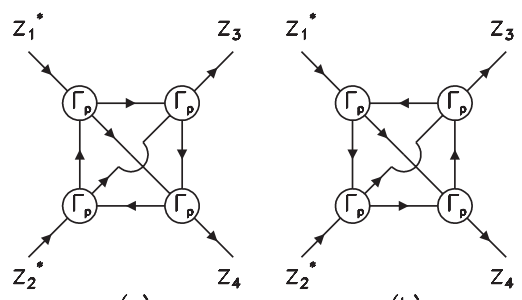

(a)

(b)

Figure 2. The leading order non-parquet diagrams. Note that a general vertex $\Gamma_{p}(\mathbf{k})$ is used on each of four vertices. There are two more diagrams obtained by exchanging $z_{3}$ and $z_{4}$.

except the bare vertex diagram. The lowest order of the non-parquet diagrams is $\mathrm{O}\left(\beta^{4}\right)$ as shown in figure 2. A straightforward way to proceed would be to calculate the non-parquet diagrams term by term starting from the fourth-order diagram. This procedure will generate a perturbation series for $R(\mathbf{k})$. To extract nonperturbative information for $R(\mathbf{k})$ and for the structure factor, one must then apply a resummation method such as the Padé approximation to the perturbation series. Such numerical resummation procedures, however, are known [10] to be less effective in capturing the growing crystalline order in the vortex liquid than the integral equation approach like the parquet approximation.

In this paper, we take a different route by incorporating an infinite subset of the non-parquet diagrams systematically into the integral equations discussed in the previous section. We first evaluate the diagrams represented by the lowest order non-parquet diagram shown in figure 2 . Note that, in place of the bare vertices, we use a general vertex $\Gamma_{p}(\mathbf{k})$ to be specified later. For any vertex $\Gamma_{p}(\mathbf{k})$, these diagrams certainly represent a subset of Feynman diagrams that is not considered in the parquet approximation i.e. that cannot be decomposed as in figure 1 . We find that there are two distinct diagrams as shown in figure 2, which turn out to give the same contribution, denoted here by $J(\mathbf{k})$. By explicitly evaluating these diagrams using a general vertex $\Gamma_{p}(\mathbf{k})$, we obtain

$$
\begin{aligned}
J(\mathbf{k})=-16 x^{3} & \left(\frac{2 \pi}{\mu^{2}}\right)^{2} \int \frac{\mathrm{d}^{2} \mathbf{p}}{(2 \pi)^{2}} \int \frac{\mathrm{d}^{2} \mathbf{q}}{(2 \pi)^{2}} \\
& \times \Gamma_{p}(\mathbf{k}-\mathbf{q}) \Gamma_{p}(\mathbf{k}-\mathbf{p}) \Gamma_{p}(\mathbf{p}) \Gamma_{p}(\mathbf{q}) \cos \left(\frac{p_{x} q_{y}-p_{y} q_{x}}{\mu^{2}}\right) .
\end{aligned}
$$

There are also contributions from the diagrams obtained by exchanging $z_{3}$ and $z_{4}$ in figure 2 , which are related to $J(\mathbf{k})$ via the hat operation defined by

$$
\hat{J}(\mathbf{k})=\frac{2 \pi}{\mu^{2}} \int \frac{\mathrm{d}^{2} \mathbf{p}}{(2 \pi)^{2}} J(\mathbf{p}) \cos \left(\frac{k_{x} p_{y}-k_{y} p_{x}}{\mu^{2}}\right) .
$$

To include the contributions from the new set of diagrams, we take in equations (5) and (6)

$$
R(\mathbf{k})=\Gamma_{B}(\mathbf{k})+J(\mathbf{k})+\hat{J}(\mathbf{k}) .
$$

Now we must specify what $\Gamma_{p}(\mathbf{k})$ is in equation (12) to close the self-consistent equations. In this paper, we take $\Gamma_{p}(\mathbf{k})$ as the sum of all the parquet diagrams, that is the solution of the parquet equations (5)-(7) when $R(\mathbf{k})$ is just $\Gamma_{B}(\mathbf{k})$ for given parameter $x$. Then the nonparquet diagrams in figure 2 represent an infinite subset of Feynman diagrams for which the skeleton diagram in figure 2 contains all the parquet diagrams on each of the four vertices. The inclusion of these contributions is achieved by solving again equations (5)-(7) but with equation (14). There are of course choices for $\Gamma_{p}$ other than the present one, which will be discussed in section 5 . We find that the present scheme is relatively easy to implement numerically and 


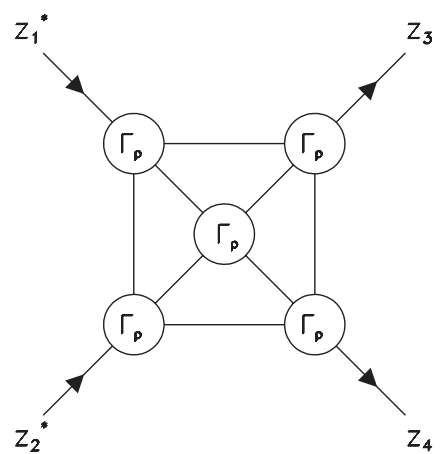

Figure 3. A higher order non-parquet diagram which can be included using the present method.

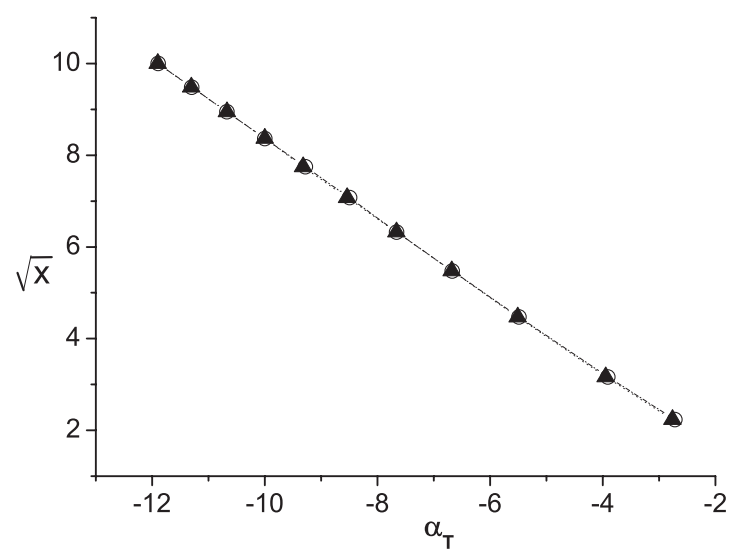

Figure 4. The renormalized propagator $\sqrt{x} \sim 1 / \alpha_{R}$ as a function of the temperature parameter $\alpha_{T}$. The filled triangles and the circles are obtained with and without the non-parquet diagrams, respectively. The dashed line is a guide for the eye.

to generalize to the next order. To get an improvement of the present approximation, we just need to calculate the contribution from the next order skeleton diagram shown in figure 3 and include it into equation (14) in a similar way.

\section{Results}

As explained in the previous section, we solve numerically the self-consistent equations (5)(7) together with (14) for $\Gamma(\mathbf{k})$. The vertex function $\Gamma_{p}(\mathbf{k})$ used in (14) is obtained by solving once equations (5)-(7) with $R(\mathbf{k})=\Gamma_{B}(\mathbf{k})$. All these numerical procedures are performed for given value of $x$. The temperature parameter $\alpha_{T}$ is determined from equation (8). Since we are considering the vortex liquid phase, we only consider a rotationally symmetric case where all the functions depend on a dimensionless momentum $K$ with $\mathbf{K}=\mathbf{k} / \mu$. The integral equations are solved numerically by iteration starting from an appropriate choice of initial $\Gamma(\mathbf{k})$. The convergence of the iteration can be improved when the solution at slightly smaller value of $x$ is used as the initial choice. We obtain the solutions for given values of $x$ up to $x=100$, which is the largest value we considered. It corresponds approximately to $\alpha_{T}=-11.9$ (see figure 4). As we go down to lower temperatures, we have to increase the $k$-space cut-off to accommodate 


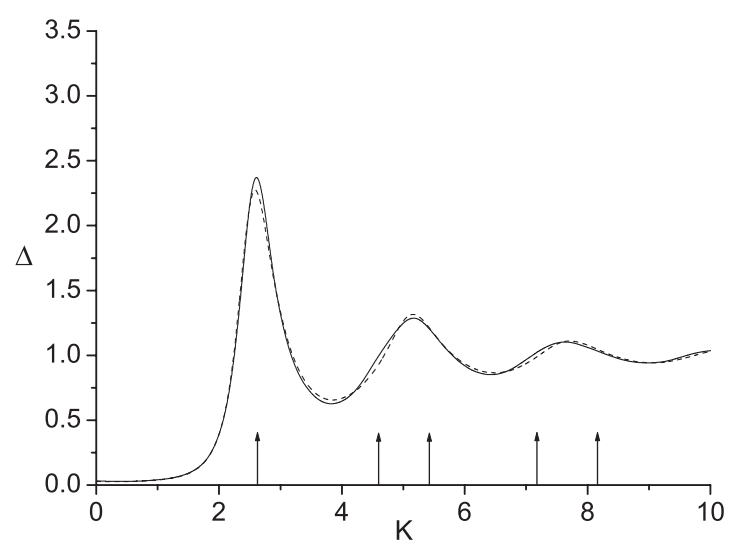

Figure 5. The structure factor $\Delta$ of the two-dimensional vortex liquid as a function of the dimensionless momentum $K=k / \mu$ at $\alpha_{T} \simeq-7.66$. The solid line is from the calculation including the non-parquet diagrams, while the dashed lines is that of the parquet approximation. The arrows indicate the positions of the RLV of the triangular lattice.

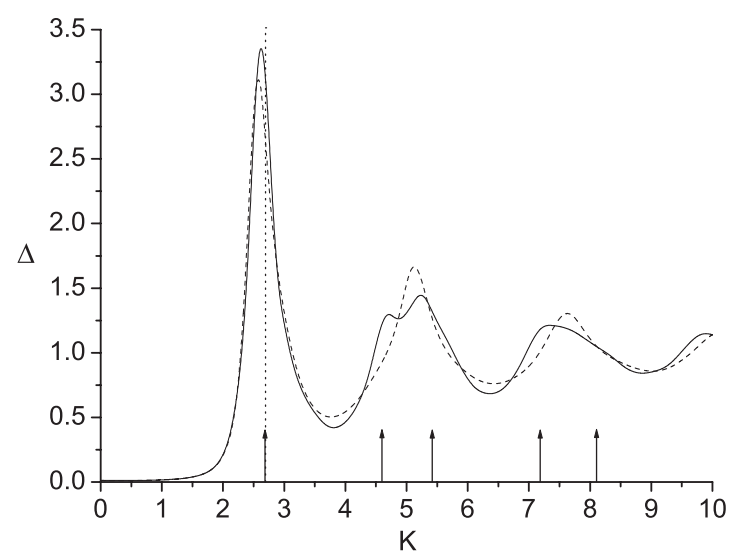

Figure 6. The structure factor at $\alpha_{T} \simeq-11.9$. The solid and dashed lines are the same as in figure 5. The expected position of the first peak from the RLV is presented as a dotted line for a guide for the eye.

the peaks appearing at large $k$ in the structure factor and decrease the grid size at the same time to capture the sharp first peak (see figures 5 and 6). The number of iterations needed to get a convergence increases as the temperature is lowered. At the lowest temperature we considered, we needed about 1000 iterations to get a convergence. All these factors limit the temperature range where the numerical solution can be obtained.

Figure 4 shows the relationship between the renormalized propagator $\sqrt{x} \sim \alpha_{R}^{-1}$ and the temperature parameter $\alpha_{T}$. The values are compared with those obtained when only the parquet diagrams are considered. We can see that there are essentially no differences between the two cases. Note that in the present analysis, we have extended the previous parquet approximation results [13] to lower temperatures $\alpha_{T} \simeq-11.9$. The dimensionless renormalized propagator $\sqrt{x}$ is directly proportional to the thermodynamic quantities like the magnetization and the entropy of the vortex liquid system. Another interesting thermodynamic quantity is the generalized Abrikosov ratio defined by $\beta_{\mathrm{A}}(x) \equiv \overline{\left\langle|\Psi(\mathbf{r})|^{4}\right\rangle} /\left[\overline{\left\langle|\Psi(\mathbf{r})|^{2}\right\rangle}\right]^{2}$, where the bar denotes 
the spatial average. As shown in [13], this quantity decreases as the temperature is lowered, and approaches 1.16, the value for a triangular lattice as $x \rightarrow \infty$ or $\alpha_{T} \rightarrow-\infty$. It is related to $\Gamma(\mathbf{k})$ in such a way that the relation (8) can be rewritten as $\alpha_{T}=\left(1-x \beta_{\mathrm{A}}(x)\right) / \sqrt{x}$. Therefore, the generalized Abrikosov ratio even in the presence of the non-parquet contributions shows behaviour similar to the result obtained in the parquet approximation [13]. From these results, we may conclude that non-parquet contributions make little difference to the thermodynamic quantities. As we will see below, however, effects of non-parquet diagrams appear in the crystalline order developing in the vortex liquid.

We calculate the structure factors (11) for the two-dimensional vortex liquid at various temperatures as shown in figures 5 and 6 . They are compared with those obtained in the parquet approximation. The structure factors for both cases look almost the same down to low temperatures $\alpha_{T} \simeq-7$. As the temperature is lowered further, however, we find that the first peak becomes slightly larger and sharper when the non-parquet diagrams are included (see figure 5). This trend continues further down to lower temperatures. When the temperature is lowered below $\alpha_{T} \simeq-10$, the second peak begins to split into two peaks (see figure 6). This can be interpreted as the non-parquet contributions capturing the growing crystalline order in the vortex liquids more effectively. The peaks developing in the structure factor correspond to the positions of the reciprocal lattice vectors (RLV) of the triangular lattice. The RLV $\mathbf{G}$ can be represented in a dimensionless form as $\mathbf{G} / \mu=G_{0}(m \eta, n-m \zeta)$ using a set of integers $m$ and $n$, where, for the triangular lattice, $\eta=\sqrt{3} / 2, \zeta=1 / 2$ and $G_{0}=\sqrt{2 \pi / \eta} \simeq 2.694$. Therefore the lengths of the RLV can be grouped into $|\mathbf{G}| / \mu=c_{i} G_{0}, i=1,2,3 \ldots$, where $c_{1}=1, c_{2}=\sqrt{3}, c_{3}=2, c_{4}=\sqrt{7}, c_{5}=3$, etc. The first peak in the structure factor is located near $K=G_{0}$. Comparing the results in figure 6 for the cases with and without the non-parquet diagrams, we can see that when the non-parquet contributions are included, the first peak is closer to its expected position. Since, for a triangular lattice, the second and third sets of RLV are relatively closely spaced, they appear as one peak within the parquet approximation. Figure 6 shows that the resolved peaks are located near the expected positions of the RLV when the non-parquet contributions are included. The situation is similar for the closely spaced fourth and fifth peaks. Although the peaks are not resolved at the minimum temperature we have studied, we can clearly see a tendency compared to the parquet results.

\section{Discussion and conclusion}

In summary we have generalized the parquet approximation for the two-dimensional vortex liquid systems by demonstrating that the non-parquet contributions can be included systematically into the nonperturbative calculation of the correlation functions. The crystalline order developing in the vortex liquid as the temperature is lowered is captured more effectively with the inclusion of the non-parquet diagrams in the sense that the peaks in the structure factor corresponding to the RLV of the triangular lattice become resolved.

In [13], within the parquet approximation, no finite temperature phase transition has been observed in the two-dimensional vortex liquid as the temperature is lowered. The length scale characterizing the growing crystalline order in the vortex liquid was determined from the width of the first peak in the structure factor. It was shown that this length scale grows like $\sqrt{x} \sim\left|\alpha_{T}\right|$ in the low temperature limit. Since the first peaks in the structure factor change only slightly with the inclusion of the non-parquet diagrams, these conclusions drawn from the parquet approximation remain valid. The detailed structure factor has a more accurate form in the present generalization.

As mentioned in section 3, there are other possible generalizations of the parquet approximation than the present one. They correspond to different choices for the vertex $\Gamma_{p}(\mathbf{k})$ 
used in equation (12). We can, for example, take $\Gamma_{p}$ equal to the full vertex $\Gamma(\mathbf{k})$ not just the sum of all the parquet diagrams. Obviously the diagrams in figure 2 in this scheme contain more diagrams than in the present one. We have attempted to solve numerically the set of equations (5)-(7) and (14) with (12) when $\Gamma_{p}=\Gamma$. At relatively high temperatures, we find there is very little difference in correlations of the vortex liquid between this scheme and the present one. But the numerical iteration in this case involves repeated evaluation of the integral in equation (12) at every step of the iteration, which considerably slows down the whole calculation. We find that it is not practical to use this approximation below $\alpha_{T} \simeq-3$. We believe that the effect of the non-parquet diagrams is already captured in the present approximation scheme.

\section{Acknowledgment}

This work was supported by Konkuk University in 2005.

\section{References}

[1] Blatter G, Feigel'man M V, Geshkenbein V B and Larkin A I 1994 Rev. Mod. Phys. 661125

[2] Abrikosov A A 1957 Zh. Eksp. Teor. Fiz. 321442 Abrikosov A A 1957 Sov. Phys._JETP 51147 (Engl. Transl.)

[3] Schilling A, Fisher R A, Phillips N E, Welp U, Dasgupta D, Kwok W K and Crabtree G W 1996 Nature 382791

[4] Kwok W K, Fendrich J, Flescher S, Welp U, Downey J and Crabtree G W 1994 Phys. Rev. Lett. 721092

[5] Liang R, Bonn D A and Hardy W N 1996 Phys. Rev. Lett. 76853

[6] Junod A, Roulin M, Genoud J-Y, Revaz B, Erb A and Walker E 1997 Physica C 275245

[7] Roulin M, Junod A, Erb A and Walker E 1998 Phys. Rev. Lett. 801722

[8] Schilling A, Fisher R A, Philips N E, Welp U, Kwok W K and Crabtree G W 1997 Phys. Rev. Lett. 784833

[9] Ruggeri G J and Thouless D J 1976 J. Phys. F: Met. Phys. 62063 Ikeda R, Ohmi T and Tsuneto T 1990 J. Phys. Soc. Japan 591397 Brézin E, Fujita A and Hikami S 1990 Phys. Rev. Lett. 651949 Hikami S, Fujita A and Larkin A I 1991 Phys. Rev. B 4410400 Li D and Rosenstein B 2001 Phys. Rev. Lett. 863618 Li D and Rosenstein B 2001 Phys. Rev. B 65024513

[10] Hu J, MacDonald A H and McKay B D 1994 Phys. Rev. B 4915263

[11] Tešanović Z and Xing L 1991 Phys. Rev. Lett. 672729 Kato Y and Nagaosa N 1993 Phys. Rev. B 472932 Hu J and MacDonald A H 1993 Phys. Rev. Lett. 71432 Š́sik R and Stroud D 1994 Phys. Rev. B 4916074

[12] O’Neill J A and Moore M A 1993 Phys. Rev. B 48374 Dodgson M J W and Moore M A 1997 Phys. Rev. B 553816

[13] Yeo J and Moore M A 1996 Phys. Rev. Lett. 761142 Yeo J and Moore M A 1996 Phys. Rev. B 544218

[14] Yeo J and Moore M A 2001 Phys. Rev. B 64024514

[15] Yeo J 1999 J. Korean Phys. Soc. 35108

[16] Hansen J-P and McDonald I R 1986 Theory of Simple Liquids (London: Elsevier Academic Press)

[17] Tešanović Z and Andreev A V 1994 Phys. Rev. B 494064 Ikeda R 1995 J. Phys. Soc. Japan 641683

[18] See for example Larkin A I and Khmel'nitskii D E 1969 Zh. Eksp. Theor. Fiz. 562087 Larkin A I and Khmel'nitskii D E 1969 Sov. Phys._JETP 291123 (Engl. Transl.)

Roulet B, Gavoret J and Nozières P 1969 Phys. Rev. 1781027

Tsuneto T and Abrahams E 1973 Phys. Rev. Lett. 30217 